\title{
Philosophiques
}

\section{Philosophiques a vingt ans !}

\section{Josiane Boulad-Ayoub}

Volume 21, numéro 1, printemps 1994

URI : https://id.erudit.org/iderudit/027272ar

DOI : https://doi.org/10.7202/027272ar

Aller au sommaire du numéro

Éditeur(s)

Société de philosophie du Québec

ISSN

0316-2923 (imprimé)

1492-1391 (numérique)

Découvrir la revue

Citer ce document

Boulad-Ayoub, J. (1994). Philosophiques a vingt ans ! Philosophiques, 21(1),

I-LXI. https://doi.org/10.7202/027272ar

Ce document est protégé par la loi sur le droit d'auteur. L'utilisation des services d'Érudit (y compris la reproduction) est assujettie à sa politique d'utilisation que vous pouvez consulter en ligne.

https://apropos.erudit.org/fr/usagers/politique-dutilisation/ 


\section{PHIIOSOPHIOUES A VINGT ANS ! 1974-1994}

Philosophiques a vingt ans! Pour souligner cet anniversaire nous sommes heureux de présenter à nos lecteurs, sous forme d'images numérisées, les vingt années de la vie de notre revue.

On trouvera par volume, les sommaires, les conseils et les comités de rédaction successifs depuis la fondation de Philosophiques, en 1974, par Monsieur Yvon Lafrance. Nous donnons aussi la présentation de la nouvelle revue dans le volume I, $\mathrm{n}^{\circ} \mathrm{I}$, de son premier directeur et fondateur.

Pas tout à fait un « Bulletin », mais plus qu'un album de famille que l'on feuillette d'u ceil attendri, les pages qui suivent représentent, de façon privilégiée, la vitalité remarquable de la communauté philosophiqued'expression française au Canada depuis quatre lustres, son activité intellectuelle comme les mouvements et les débats qui l'animent. En résonance avec sa culture propre et ses enjeux symboliques ou sociaux, sa voix, de manière distinctive, s'harmonise avec le concert de la communauté scientifique universelle. Voici ce que nous révèle ces quarante sommaires de «l'organe officiel de la Société de philosophie du Québec ».

Josiane Boulad-Ayoub 5 directrice de Philosophiques 


\section{AVRLL 1974}

\section{Rédaction}

Faculté de Philosophie de l'Université d'Ottawa

90, rue Wilbrod, Ottawa KIN 6NS

Ontario, Canada

Directeur : Yvon Lafrance

Assistant-Directeur : Roch Bouchard

Comité de Rédaction: J. Croteau, B. Garceau, Guy Lafrance, G. Charron, P. McCormick

\section{Conseil de la Reyue}

V. Cauchy (Universiry de Montréal), E. Trépanier (Université Laval)

C. Savary (Université du Québec à Trois-Rivières) N. Lacharité

(Université du Québec à Montréal, J. Plamondon (Université de Sherbrooke),

P. Laberge (Université d'Ortawa), S. Morin (Université de Moncton),

V. Berens (Université de Sudbury) 


\section{SOMMAIRE}

Volume 1 , numéro 1

Avril 1974

Présentation

1. ARTICLES

M. GUÉROULT, Méthode en histoire de la philosophie 7

B. GARCEAU, Les travaux de jeunesse de Hegel et l'interprétation de sa philosophie de la religion ....

M. LAGUEUX, L'arrière-fond philosophique du concept de plan

Y. GAU'THIER, Constructivisme et structuralisme dans les fondements des mathématiques

G.B. MADISON, Le postulat d'objectivité dans la science et la philosophie du sujet

Résumés des articles — Abstracts of the Articles

2. ÉTUDES CRITIQUES

S. AUROUX, L'empirisme de Locke, par F. Duchesneau 147

3. INTERVENTIONS

S.J. MORIN, Sur l'injustice de structure

4. CHRONIQUE

Congrès de l'ACFAS et de l'ACP. La Société de Philosophie de Montréal. La Société de Philosophie du Québec. Cercle de philosophie de Trois-Rivières. Activités des Facultés et Départements de Philosophie 


\section{PRÉSENTATION}

La fondation d'une nouvelle revue provoque souvent des sentiments d'enthousiasme mêlés de scepticisme. Pourquoi une nouvelle revue philosophique alors qu'il en existe de si nombreuses et parfois fort excellentes?

Chaque fondation de revue obéit à des motifs et à des objectifs les plus divers inscrits dans le milieu même qui la voit naître. Le dynamisme qui se propage depuis quelques années dans les milieux philosophiques d'expression française au Québec et au Canada tendait naturellement à la naissance d'une nouvelle revue. En effet, la jeune génération de professeurs de philosophie dans nos collèges et universités éprouve de plus en plus le besoin de prolonger son enseignement dans des activités de recherche et de publication. Cette jeune génération a compris que la présentation de ses travaux au grand public philosophique constitue un moyen nécessaire et indispensable pour améliorer sa compétence professionnelle. La revue se veut un instrument de travail pour ces jeunes énergies qui cherchent à s'exprimer et qui, en son absence, auraient de la difficulté à le faire. En effet, Philosophiques est présentement la seule revue entièrement d'expression française au Canada, consacrée exclusivement à la philosophie. Cette unique remarque suffirait à justifier la pertinence de sa fondation.

Le titre de la revue Philosophiques est déjà tout un programme. Par sa désinvolture grammaticale, le titre souligne la volonté de progrès et d'ouverture à toutes les idées philosophiques. La rubrique «Interventions» sera consacrée à des articles à caractère moins académique et plus engagé et voudrait être le laboratoire des modes d'expression nouveaux, là où l'esprit créateur ne craint pas de s'engager à ses risques et dépens. Il nous apparait, en effet, essentiel pour le dynamisme de nos milieux philosophiques, que des esprits puissent s'engager en dehors 
des sentiers battus et des valeurs sûres pour frayer des voies nouvelles à la créativité philosophique. C'est la marge de risque que le comité de rédaction assume en toute lucidité.

Le titre ne souligne pas seulement une volonté de progrès dans la création philosophique, mais aussi une politique fondamentale d'ouverture à tous les courants philosophiques. En calquant le vieil adage bien connu, nous dirions que rien de vraiment philosophique ne saurait lui être étranger. La revue se veut franchement le carrefour soumis à tous les vents des écoles philosophiques sans discrimination d'aucune. Les chercheurs, nous l'espérons, trouveront toujours dans la revue un esprit inébranlable de tolérance à l'égard de toutes les idées philosophiques. Le seul critère utilisé dans l'approbation des manuscrits sera celui de la qualité professionnelle. C'est pourquoi, pour garantir cette qualité professionnelle, avons-nous cru bon de publier sur la couverture intérieure de la revue notre procédure officielle d'approbation. Ceci dit, la rubrique «Articles » sera consacrée à la publication des travaux de recherche en philosophie tandis que la rubrique «Etudes critiques» essaiera de faire la promotion pour un public national et international d'ouvrages de philosophie rédigés par des professeurs travaillant dans une institution canadienne. Nous espérons par là encourager les jeunes talents à publier.

Organe de recherche et de discussion, la revue Philosophiques veut être aussi un organe d'information. A cette fin, nous aurons la rubrique «Chronique» qui se chargera de renseigner le lecteur sur les activités de nos milieux philosophiques d'expression française au Canada et au Québec. Cette chronique pourrait être également utile au lecteur étranger de plus en plus curieux à l'égard de la philosophie au Canada. De plus, nous croyons qu'au fil des années, cette chronique pourrait devenir une riche documentation aux mains des historiens de la philosophic au Québec et au Canada.

Deux rubriques ne figurent pas dans le sommaire de ce premier numéro, mais apparaîtront, nous l'espérons, dans les prochains numéros. Il s'agit du «Bulletin» et du "Compte Rendu». Ia première rubrique se propose d'informer le lecteur sur l'état actuel de la recherche dans un domaine particulier de 
la philosophie. Les premiers bulletins seront surtout consacrés à l'état de la recherche en philosophie au Québec et au Canada et pourraient devenir ainsi le point de départ pour de nouvelles orientations de recherche. Quant à la seconde rubrique, elle informera le lecteur sur les productions de première valeur en philosophie, au niveau national et international.

Organe de recherche et d'information, véhicule sympatique des nouvelles expressions créatrices de la pensée philosophique, la revue, nous l'espérons, ne pourra que refléter et stimuler le dynamisme de nos milieux philosophiques d'expression française au Canada.

En terminant, on nous permettra de remercier tous ceux qui depuis deux ans ont travaillé à la fondation de cette revue: l'ancien doyen de la Faculté de Philosophie de l'Université d'Ottawa, le P. Gilles Cazabon ainsi que le doyen actuel, le Prof. Pierre Laberge, les membres du comité de rédaction, les doyens des Facultés et les directeurs des départements de philosophie ainsi que le Conseil des Arts du Canada dont la subvention substantielle a permis la réalisation de ce projet.

Et maintenant, lecteur, il ne nous reste plus qu'a larguer les amarres! Et que le vent souffle au gré de la déesse Vie, source toujours intarissable d'inspiration féconde!

Yvon Lafrance

Directeur 


\section{OCTOBRE 1974}

Ia revue "Philosophiques, est publiée gràce à une subvention du Conseil des Arts du Canada et avec l'aide financière des Facultés et Départements de Philosophie ì"expression française au Canada.

Elle parait deux fois par année, en avril et en ocrobre.

\section{CONSEIL DE LA REVUE}

E. Trépanier (Universiré Laval)

J.P. Audet (Université de Montríal)

N. Lachariti (Universite du Qućbec à Montríal)

C. Savary (Université du Québec à Trois-Rivièris's)

J. Bachand (Université du Qućbec à Chicoutimi)

J. Plamondon (Université de Sherbrooke)

P. Laberge (Université d'Otrawa)

R. Champagne (Université de Sudbury)

G. François (Université de Moncton)

P.A. Quintin (Président de la Sociéré de Philosophie du Québec)

\section{RÉDACTION DE LA REVUE}

Directeur: Yvon Lafrance (Otuawa)

Assistant-Directeur :Roch Bouchard (Ottawa)

Comité de rédaction: Raymond Brouillet (Layal)

Benoît Garceau (Otrawa)

Robert Nadeau (UQAM)

Jacques Croredu (Ortawa)

Maurice Lagueux (Montréal)

Guy Lafrance (Otcawa)

N. Kaufmann (Saint-Laurent)

Ghyslain Charron (Oteawa)

Peter McCormick (Ottawa) 


\section{SOMMAIRE}

Volume 1, numéro 2

Octobre 1974

1. ARTICLES

L. VALCKE, Le monisme épistémologique de la science contemporaine

P. McCORMICK, Heidegger sur le chemin du langage

C. PANACCIO, Langage ordinaire et langage abstrait chez Guillaume d'Occam

G.-H. ALLARD, L'énigme et la culture littéraire d'Augustin

Résumés des articles - Abstracts of Articles

2. ETUDES CRITIQUES

O. REBOUL, La théologie kantienne pré-critique, par

P. Laberge

D. SAUVE, La cohérence de la doctrine kantienne de la liberté, par B. Carnois

3. BULLETIN

A.M. LANDRY, O.P., La pensée philosophique médiévale. Contribution canadienne (1960-1973)

4. INTERVENTIONS

J. D'HONDT, Utopie et Liberté

5. CHRONIQUE

Congrès. La Société de Philosophie du Québec. La Société de Philosophie de Montréal. Activités des départements de philosophie. Publication et instruments de travail. 
F. Trépanier (Université Laval)

J.P. Audet (Université de Montréal)

N. Lacharité (Université du Québec à Montrial)

C. Savary (Université du Québec à Trois-Rivières)

J. Bachand (Université du Québec à Chicoutimi)

J. Plamondon (Université de Sherbrooke)

P. Laberge (Université d'Ottawa)

R. Champagne (Université de Sudbury)

G. François (Université de Moncton)

P.A. Quintin (Président de la Société de Philosophie du Québec)

\section{RÉDACTION DE LA REVUE}

Directeur: Yvon Lafrance (Otawa)

Assistant-Directeur :Roch Bouchard (Ottawa)

Comité de rédaction: Raymond Brouillet (Laval)

Benoit Garceau (Ottawa)

Robert Nadeau (UQAM)

Jacques Croteau (Otrawa)

Maurice Lagueux (Montréal)

Guy Lafrance (Ottawa)

N. Kaufmann (Saint-Laurent)

Ghyslain Charton (Ottawa)

Peter McCormick (Ottawa)

CONSEIL DE LA REVUE

Th. De Koninck (Université Laval)

J.P. Audet (Université de Montréal)

H. Malouin (Universitć de Québec à Montríal)

C. Savary (Université du Québec à Trois-Rivières)

J. Bachand (Universicé du Québec à Chicoutimi)

M. Gagnon (Universite de Sherbrooke)

P. Laberge (Universié́ d'Ottawa)

R. Champagne (Université de Sudbury)

G. François (Université de Moncton)

P.-A. Quintin (Président de la Société de Philosophie du Qućbec)

\section{RÉDACTION DE LA REVUE}

Directeur : Yvon Lafrance (Ottawa)

Comité de rédaction:

Benoit Garceau, responsable du \& Bulletin > (Ortawz)

Robert Nadeau, responsable de e Interventions s (UQAM)

Raymond Brouillet (Laval)

Maurice Lagueux (Montréal)

Guy Lafrance (Ottawa)

Nicolas Kaufmann (UQTR)

Ghyslain Charron (Ortawa)

Peter McCormick (Otrawa) 


\section{SOMMAIRE}

Volume II, numéro 1

Avril 1975

1. ARTICLES

J. THEAU, Le rapport quantité-qualité chez Hegel et chez Bergson

A. VACHET, La dialectique de l'individu et de la collectivité dans la pensée de Marx

L.-P. BORDELEAU, Une genèse de la vie sociale selon Maurice Blondel

S. VASSILIE-LEMENY, Structure et méthode dans la philosophie du sens et de la valeur

2. ÉTUDES CRITIQUES

G.B. MADISON, Vers une nouvelle philosophie transcendantale, par Th. F. Geraets

TH. F. GERAETS, La Phénoménologie de MerleauPonty, par G.B. Madison

Réponses des auteurs

3. BULLETIN

R. HÉBERT, Introduction à l'histoire du concept de réflexion: position d'une recherche et matériaux bibliographiques

4. INTER VENTIONS

M. PATRY, La fonction critique de l'Université

5. CHRONIQUE

La Société de Philosophie du Québec. La Société de Philosophie de Montréal. Le Cercle de Philosophie de Trois-Rivières. La Société de Philosophie de l'Outaouais. Activités des Départements. Publications. 


\section{SOMMAIRE}

Volume II, numéro 2

Octobre 1975

1. ARTICLES

M. GAGNON, Une analyse sémantique du concept de causalité est-elle possible?

L. GIROUX, Heidegger et la Métaphysique : vers un double dépassement

P. McCORMICK, L'esthétique de Dilthey : Phénoménologie et théorie littéraire

2. ETUDES CRITIQUES

G. LEROUX, Platon. La Médiation du Regard, par L. Paquet

J. TROUILLARD, Le Même et l'Autre dans la structure ontologique du «Timée», de Platon, par L. Brisson

3. BULLETIN

B. GARCEAU, La philosophie analytique de la religion: contribution canadienne (1970-1975)

4. INTER VENTIONS

Autour de la Loi 50

R. NADEAU, Présentation

G.-A. LEGAULT, Législation et droits de l'homme

L. MARCIL-LACOSTE, Quand l'État devient philosophe

C. PANACCIO, Le projet de loi 50 et la place des droits de l'homme dans le système juridique

5. CHRONIQUE

Congrès. La Société de Philosophie du Québec. Le Cercle de Philosophie de Trois-Rivières. La Société de Philosophie de l'Outaouais. Activités des Départements.

Publications et instruments de travail 


\section{CONSEIL DE LA REVUE}

Th. De Koninck (Université Laval)

J.P. Audec (Université de Montréal)

H. Malouin (Universitć de Québec à Montrćal)

C. Savary (Université du Québec à Trois-Rivières)

J. Bachand (Université du Québec à Chicoutimi)

M. Gagnon (Université de Sherbrooke)

P. Laberge (Université d'Ottawa)

R. Champagne (Université de Sudbury)

G. François (Université de Moncton)

P.-A. Quintin (Présidenc de la Société de Pltilosophie du Québec)

\section{RÉDACTION DE LA REVUE}

Directeur : Yvon Lafrance (Ottawa)

Comitć de rédaction:

Benoît Garceau, responsable du * Bulletin s (Ottawa)

Robert Nadeau, rcsponsable de anterventions \(UQAM)

Raymond Brouillet (Laval)

Maurice Lagueux (Montréal)

Guy Lafrance (Ottawa)

Nicolas Kaufmann (UQTR)

Ghyslain Charron (Ottawa)

Peter McCormick (Ottawa) 


\section{SOMMAIRE}

Volume III, numéro 1

Avril 1976

I. ARTICLES

J.N. KAUFMANN, Structure et causalité

M. RENAULT, Nouvel essai sur les sandales d'Empédocle

Y. LAMONDE et B. LACROIX, Les débuts de la philosophie universitaire à Montréal. Les Mémoires du doyen Forest, O.P. (1885-1970)

2. ÉTUDES CRITIQUeS

J. GOULET, La philosophie sociale de Bergson, Sources et incerprétation, par Guy Lafrance

3. INTERVENTIONS

\section{PREMIĖRE PARTIE}

La philosophie institutionnelle: critique et/ou idćologie?

R. NADEAU, Présentation

I. J.-G. DAOUST, Endoctrinement et politisation dans l'enseignement de la philosophie ou d'un faux problème

II. J. PROULX, Par-delà l'idéologie et le soupçon : l'authenticité intellectuelle

III. J.-P. BRODEUR, Sur les justifications de la philosophie institutionnalisće

DEUXIÈME PARTIE

D. BRAYBROOKE, $\grave{A}$ la recherche d'une justice de structure au Nouveau-Brunswick

4. CHRONIQUE

La Société de Philosophie du Québec. La Société de Philosophie de l'Outaouais. Le Cercle de Philosophie de Trois-Rivières. Activités des Départements. Publications. 


\section{SOMMAIRE}

Vol. III, numéro 2

Octobre 1976

1. ARTICIES

F. DUCHESNEAU, Sémiotique et Abstraction : de Locke à Condillac

J. CARON, Dialectique de la Communication chez Kierkegaard.

J. THEAU, Remarques sur l'épistémologie française et l'épistémologie américaine.

J.-P. BRODEUR, De l'orthodoxie en philosophie

2. ÉTLIDES CRITIQLIES

P. MARTIN, Du langage. A. Martinet et M. Merleau-Ponty, par G. Charron.

G. CHARRON, Linguistique, Philosophie du langage et Épistémologie (Réponse à P. Martin)

G. LAFRANCE, Lire Bergson (Réponse à J. Goulet)

3. BULLETIN

J. KING-FARLOW, L'immutabilité du Dieu biblique et du Deus Thomisticus (Réponse à B. Garceau).

4. INTERVENTIONS

L. CHARETTE. L'idéologie dans l'éducation

5. (HRONIQLIE

La Société de Philosophie du Québec. La Société de Philosophie de Montréal. La Société de Philosophie de l'Outaouais. La Société de Philosophie de Sherbrooke. Activités des Départements. Publications. 


\section{CONSEIL DE LA REVUE}

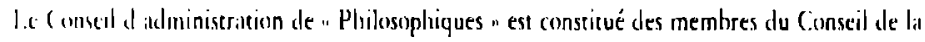
Sincéti: we Philemsphe du Quéhect

\section{RÉDACTION DE LA REVUE}

Directeur: (jult Bouchard (Québec)

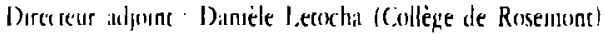

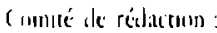

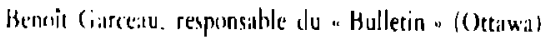

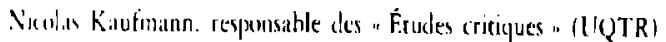

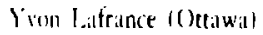

Maurrice Ciagnen (Sherhronke)

Milures I.ilgutux (Moneréal)

(icurges l.ctoux (l:QAM)

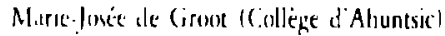

C.lude Cingnen If nollege lidouird-Monteperis!

Sherela Mullet is.oncordul 


\section{SOMMAIRE}

Vol IV, numéro 1

Avril 1977

1. ARTICLE

W. H. DRAY, Les explications causales en histoire.... 3

2. ÉTUDE CRITIQUE

G. CHARRON, Hobbes et Freud, par Jean Roy ...... 35

3. BULLETIN

Y. LAFRANCE, Les Études platoniciennes : contribution canadienne (1970-1977)

4. INTERVENTIONS

L'enseignement de la morale et la question de ses fondements

R. NADEAU, Présentation .................... 101

1. L. DUCHARME, Morale objective et loi naturelle 102

2. R. NADEAU, L'enseignement de la morale dans une perspective relativiste .................109

3. E. JOOS, Le fondement de la morale et l'enseignement de la morale

4. L. MARCIL-LACOSTE, Le monopole confession. naliste dans son rapport avec la réflexion sur l'Éthique ............................. 125

5. B. PRUCHE, Discussion ................. 136 


\section{SOMMAIRE}

Vol. IV, numéro 2

Octobre, 1977

NADEAU, R.,

Liminaire

NOELTING, G.,

Le constructivisme piagétien et la théorie de l'équilibration illustrés par la construction de la notion de proportion

BRIEF, J.-C.,

Le constructivisme piagétien et les épistémologies traditionnelles

GAGNON, M.,

Épistémologie génétique, science et philosophie

PLANTE, R.,

Le phénomène cognitif comme éco-système chez Piaget 245

STERLIN, C.,

Les contestations contemporaines de la psychiatrie : de

l'antipsychiatrie à la néo-psychiatrie

SAVOIE, R.,

La psychiatrie : une machine à faire croire

LAFERRIEkE, $M$.,

Les pièges de l'antipsychiatrie

LÉVESQUE, C.,

L'inscription de la psychanalyse

PERALDI, F.,

La psychanalyse nord-américaine

CHARRON, G., Inconscient social de la psychanalyse et points aveugles du psychanalyste

MONETTE, L.,

Le processus contre-transférentiel comme travail de deuil 305

KAUFMANN, J.N.,

Psychologie de la " conscience " et science du " behavior " 313

SCHLEIFER, M.,

Behaviorisme et psychologie

REINBOLD, J.,

Le behaviorisme et les psychologues sociaux 


\section{CONSEIL DE LA REVUE}

le Consell dadministration de "Philosophiques " tst constirué ses membres du Conseil de his Société de Philusaphue du Quéhet

\section{COMITE DE REDACTION DE LA REVUE}

Dirtuleur . (ius Bouchard (Québec)

Diresteur adjornt: Daniele Letocha IRusemont!

Comuté de rédaction :

Henoit Girceatu, responsahle du * Bulletin " (Otrawa)

Ywon Lafrance, responsable des * Érudes cririques - (Otcawa)

Maurice (jagnom (Sherhroke)

Misure lagueux IMonertal

Cieorges leroux IL'QAM

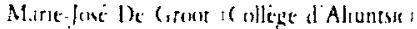

( haude (iignon (Colleget Edouard-Montpent)

Sheela Multere (concordia) 


\section{SOMMAIRE}

Vol. V, numéro 1

Avril 1978

1. ARTICLES

J. BÉLANGER, Images et réalités du behaviorisme.. 3

P. GRAVEL, Pour une logique de l'action tragique : Hegel et la tragédie ...................... 111

M. HENRY, Qu'est-ce que cela que nous appelons la vie ? ............................... 133

R. HOUDE, $\AA$ propos (Réflexions) ............ 151

2. ÉTUDES CRITIQUES

C. PANACCIO, Comment parler de la littérature? par Raymond Montpetit....................... 155

R. MONTPETIT, Réponses au commentaire de Claude Panaccio sur Comment parler de la littérature? .. 173

3. INTERVENTIONS

1. Les "interdictions philosophiques" J. BOULAD AYOUB, Présentation ............ 179

P. RAYMOND, Les “ interdictions philosophiques " 181

P. RAYMOND, Réponse à Yvon Gauthier ...... 189

Y. GAUTHIER, Réplique à Pierre Raymond .... 192

2. F. COUTURIER, Le pays de la culture ....... 195

3. G. LEROUX, La recherche et le comité de déontologie à l'université 200 


\section{SOMMAIRE}

Vol. V, numéro 2

Octobre 1978

1. ARTICLES

R. MONTPETIT, L'esthétique de Rodolphe de Repentigny et la phénoménologie ..............

J.N. KAUFMANN, Contributions de l'école de Francfort à la " théorie " des idéologies...

J. LEROUX, Concept de théorie et contexte diachro-

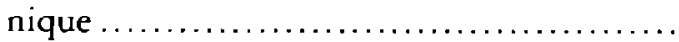

N. KATTAN, Ontologie, esthétique et œuvre d'art littéraire.............................. 261

Y. LAFRANCE, Aristote et l'analyse géométrique... 271

2. ÉTUDES CRITIQUES

A. GOMBAY, La structure performative du langage juridique par Georges Legault ..............

G.-A. LEGAULT, Réponses au commentaire de $\mathbf{M}$. André Gombay sur La structure performative du langage juridique .......................

3. BULLETIN

P. LABERGE, Dix années d'études canadokantiennes (1968-1978)

4. INTER VENTIONS

M. BÉDARD, Philosophie et culcure générale

R. PELLERIN, Réflexion et responsabilités sociales. 


\section{COMITÉ DE RÉDACTION DE LA REVUF}

Maurice Gagnon (Universiré de Sherbrooke), directeur et responsable de la rubrique * Articles".

Josiane Boulad-Ayoub (Université du Québec à Montréal), directeur adjoint et responsable de la promorion de la Revue.

Georges Leroux (Universiré du Québec à Montréal), responsable de la tubrique * Études critiques *.

Pierre Laberge (Université d'Ortawa), responsable de la rubrique * Bulletin *

André Paradis (Université du Québec à Trois-Rivières), responsable de la rubrique "Intervenrions $n$.

François Tournier (Université du Québec à Montréal), secréraire du Comité.

Guy Bouchard (Université Laval).

Stanley French (Universiré Concordia).

Maurice Lagueux (Université de Montréal).

Danièle Letocha (Collège de Rosemont).

Jean Theau (Université d'Otrawa). 


\section{SOMMAIRE}

Vol. VI, numéro 1

Avril 1979

1. ARTICLES

J. ROY, Modernité et utopie 3

C. PANACCIO, Des phoques et des hommes........ 45

F. TOURNIER, L'explicitation d'un concept ........ 65

2. ÉTUDES CRITIQUES

S. ROBERT, Fondements des mathématiques : introduction à une philosopbie constructiviste, et Méthodes et concepts de la logique formelle, par Yvon Gauthier. 119

J.-P. BRODEUR, Mobiles du discours philosophique: recherche sur le concept de réflexion, par Robert Hébert.

3. BULLETIN

S. PLOURDE, Présence de la pensée de Gabriel

Marcel au Canada (1940-1978)

4. INTER VENTIONS

M.-J. de GROOT, Présentation.

G. PARADIS, La Documentation en Philosophie : bibliographies courantes

J.-P. BRODEUR, Se taire, dit-il ? 


\section{SOMMAIRE}

Vol. VI, numéro 2

Octobre 1979

\section{ARTICLES}

G. RAULET, L'histoire guérie du concept par l'imagination. Place et fonction de l'imagination dans le marxisme contemporain........... 211

J. PESTIEAU, Société et politique avec ou sans État. 235

M. GUERTIN, L'orientation socialiste dans la philosophie politique de Shelley .................. 253

J. LEROUX, $\grave{A}$ propos de l'explicitation d'un concept ......................................... 273

F. TOURNIER, Notes sur l'explicitation d'un concept (Réponse à Jean Leroux) ............. 283

\section{2. ÉTUDES CRITIQUES}

M. GAGNON, La comparaison interculturelle, par Roberro Miguelez.

L.-M. VACHER, Marxisme et pays socialistes, par Jean-Marc Piotte.............................. 309

J.-M. PIOTTE, Note ........................... 324

3. BULLETIN

Y. LAMONDE, Lhistoire de la philosophie au Canada français (de 1920 à nos jours) : Sources et thèmes de recherche

4. INTERVENTIONS

R. HOUDE, La référence n'est pas à l'index ( $\mathrm{Sr}$ Thomas aujourd'hui) ..................... 341

J.-G. MEUNIER, Le livre blanc de "La politique québécoise du développement culturel ". Esquisse critique d'une philosophie de la culture. 347

5. HOMMAGE

M. GAGNON, Présentation....................... 361

J. GOULET, Essai de métaphysique (Nores et jalons). 


\section{SOMMAIRE}

Volume VII, numéro 1

Avril 1980

1. ARTICLES

R. TREMBLAY, L'auto-méditation phénoménologique pour une communauté des philosophes..... 3

M. GAGNON, La critique piagétienne de l'apriorisme attaque-t-elle le criticisme kantien?...... 41

L. -P. LUC, La théorie hégélienne du savoir ......... 55

2. ÉTUDES CRITIQUES

B. ROY, Claude Gagnon : de la cabale des philosophes à la lictérature des cabalistes ............ 77

J. BOULAD-AYOUB, De l'idéologie dominée, par Bernard Labrousse .......................... 85

R. HÉBERT, Histoire et pbilosopbie anl Québer. Anaribéolngie du savoir bistorique, par Roland Houde.. 93

3. INTERVENTION

G.-A. LEGAULT, La rhétorique des discours sur l'indépendance : l'argumentation historique .....101 


\section{SOMMAIRE}

Volume VII, numéro 2

Octobre 1980

1. ARTICLES

P. GRAVEL, Métaphore-Carharsis-Aufhebung

G. BOUCHARD, Le recours à l'auditoire universel implique-t-il une pétition de principe ?.........161

L. GIROUX, La connaissance historique via l'interliaison psychique : Wilhelm Dilthey.

2. QU'EST-CE QUE PROUVER SCIENTIFIQUEMENT? (Colloque tenu à l'Université du Québec à Trois-Rivières, du 10 au 12 octobre 1979).......211

C. PANACCIO, Présentation

R. NADEAU, Problématique de la preuve en épistémologie contemporaine

N. KAUFMANN, Les trois niveaux de la preuve dans les sciences empirico-formelles.

C. SAVARY, Les sciences humaines et l'interprétation..................................... 267

R.L. HOULD et M.-A. PROVOST, Éthologie et cybernétique : leur approche à la psychologic...301

3. ÉTUDES CRITIQUES

G. BOUCHARD, L'a,b,c de la sémiologie. À propos de Silence, on parle : introduction à la sémiotique, par Jurgen Pesot.

L. MONETTE, D'une lecture coupable de tout ce qu'elle laisse choir . . . À propos de Freud et le problème de la culpabilité, par Ghislain Charron ...377

4. BULLETIN

L. GIROUX, Le colloque sur “ La philosophie de l'histoire et la pratique historienne aujourd'hui ", renu à l'Université d'Ottawa du 18 au 20 avril 1980 


\section{COMITÉ DE RÉDACTION DE LA REVLE}

Maurice (iagnon (Universire de Sherbrekke), directeur et responsable de la rubreque "Arricles ".

Josiane Boulad-Ayoub (Unuversice du Quebec a Monereial), direcreur adjoint et responsable de la promorion de la Revue.

Georges Leroux (Universite du Québce a Montréal), responsalle des rubriyues "Frudes critiques " et "Compres rendus"

Picre Laberge (Université d'(Otcawa), responsable de la rubrique "Bulletın ".

Andre Paradis (Unwersitci du Quebec à Trois-Rivières), responsible de la rubrique "Interventoms $"$

Guy Bouchard (Universice Laval)

Daniele Lerocha (Uniscrsité doctawa).

Gcorges Hélal (Université de Montréal).

Rolvert Héberr (College Maisonneuve).

Stanley French (Universitci Concordia). 


\section{SOMMAIRE}

Volume VIII, numéro 1

Avril 1981

1. ARTICLES

J. ROY, Millénarisme et situationnisme ............ 3

R. PELLERIN, Théories et pratiques de la désaliénation 49

M. CARIGNAN, L'éternel comme tiers synthétisant chez Kierkegaard............................... 75

A. CLAYBOROUGH, la grammaticalité des textes litréraires................................... 93

L. CHARETTE, Droit naturel et droit positif chez saint Thomas d'Aquin

2. ÉTUDES CRITIQUES

Claude GAGNON: Préface au Speculum Maius de Vincent de Beauvais: réfraction et diffraction, par Serge Lusignan ......................................... 131

Robert HÉBERT: Rationality To-day/La vationalité aujourd'hui. Édité par T.G. Geraets.

3. INTERVENTIONS

C. COLLIN, Cricique d'une critique. Réponse à Louise Marcil-Lacoste.

V. DI NORCIA, Le fédéralisme, l'État et la démocratie 167

M. CHABOT, Le philosopbe chat ou les ruses du désir, de Roger Savoie.

4. COMPTES RENDUS

LuC BRISSON, Platon 1958-1975 (Georges Leroux) .... 197

EN COLLABORATION, Scolastique, certitude et recherche; en hommage à Louis-Marie Régis (Claude Gagnon)..... 199

Jean ROY, Hobbes et Freud (Bela Egyed) .............. 202

Jim MaCadaM, Michaël Neuman et Guy LaFRanCE, Trent Rousseau Papers; Études Rousseau-Trent (Josiane Boulad Ayoub) ........................... 204

Jean-Louis A LLARD, L'éducation à la liberté, ou la philosophie de l'éducation de Jacques Maritain (André Vidricaire)

Paul-Marcel LEMAIRE, Les signes sauvages, Une pbilosophie de langage ordinaire (François Latraverse) ......... 209

Julien BIGRAS, Le choc des oeuvres d'art Josiane Boulad Ayoub) ..................................... 211

EN COllaboration, Philosophers Look at Canadian Confederation/La Confédération canadienne: qu'en pensent les philosophes? (Robert Héberr).............. 214

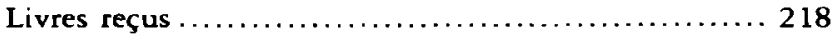




\section{SOMMAIRE}

Volume VIII, numéro 2

Octobre 1981

ARTICLES

P. McCORMICK, Sur le développement du concept de l'intentionnalité chez Brentano et Husserl................................... 227

C. PANACCIO, L'intentionnalité comme phénomène linguistique.... 239

J. PESTIEAU, Le pouvoir de l'idéal et l'idéal du pouvoir........... 259

Y. GAUTHIER, Sur la théorie des démonstrations ............... 273

A. PARADIS, Individus ou structures; existe-t-il une étbique marxiste? 287

M. BELLEFLEUR, Travail et loisir. Du loisir antique au loisir contemporain

\section{ÉTUDE CRITIQUE}

P. THIBAULT. L'bistoire du thomisme québécois ou De l'importance de mettre les ames à leur place.

\section{COMPTES RENDUS}

Julien J. LAFONTANT. Mantesquieu et le probleme de l'esclavage dans L'Esprit des lois, par Josiane Boulad Ayoub.... 349

Charles MURIN, Nietzsche probleme, généalogie d'une pensée, par Joseph Tchao....................................

Jacques J. RUELLAND, Bibliographie des oeuvres de Gaston Bachelard, ainsi que des divers ouvrages que sa pensée et sa personne ont inspirés, par Maurice Gagnon

Chiara CRISTIANI et Claude GAGNON, Alchimie et philosopbie au moyen âge, par Louis-Claude Paquin .............. 356

Pierre GRAVEL, Pour une logique du sujet tragique. Sopbocle, par Guy Bouchard ................................ 358

Guy MASSICOTTE, L'bistoire probleme. La méthode de Lucien Febore, par François Tournier.

William DRAY, Perspectives an History, par Robert Nadeau. 363

LIVRES REÇUS 


\section{SOMMAIRE}

Volume IX, numéro 1

Avril 1982

\section{ARTICLES}

G. MASSICOTTE, Critique de la pensée hiscorique. Les premiers historiens français du mouvement ouvrier

D. LETOCHA, Le trop dir, le dit, le mal dit et le non dit dans le discours althussérien sur l'idéologie

P. GRAVEL, Hegel et la construction de l'État. Contribution à sa destruction.

L. -P.LUC, Le concept de démocratie dans la critique du droit politique hégélien.

Y. GAUTHIER, Vérité et vérification en logique mathématique et dans les théories physiques

\section{BULLETIN}

A. MCKINNON, Les érudes kierkegaardiennes au Canada.

INTER VENTION

G. RAULET, L'appel de l'histoire. La théorie critique de l'école de Francfort face au contexte français

COMPTES RENDUS

E.L. FORTIN, Dissidence et philosopbie au moyen-âge. Dante et ses antéédents, par Claude Gagnon....

Benoît PRUCHE, Existant et acte d'être. Essai de philosophie existentielle, par Jourdain Lavoie ......................... 178 


\section{SOMMAIRE}

Articles

G. BOUCHARD, Gilson, l'oeuvre d'art et le roman. ........ 195

F. DUCHESNEAU, Leibnitz et les bypothèses de physique.

J.J. COURTINE, Définition d'orientations théoriques et construction de procédures en analyse du discours. . . . . . . . . . . . 239

P. BELLEMARE, Symbole: fondements anthropobiologiques de la doctrint aristotélicienne du langage. .................... 265

Érudes critiques

R. HÉBERT, D'une falaise d'où l'on voit poindre le soleil de la culture savante. (Le premier cahier de l'Institut Québécois de Recher-

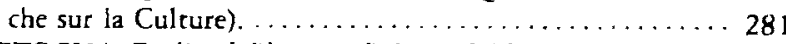

D. LETOCHA, Du lieu de l'bomme à l'absence de l'bomme. (L'antbropoIogie en l'absence de l'bomme, de Fernand Dumont).

\section{Interventions}

L. ARMOUR, Religion et philosophie au Québec et au Canada anglais. (La philosopbie et son enseignement au Québec, 1665-1920,

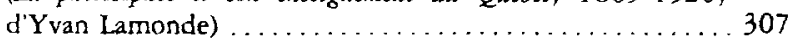

G.A. LEGAULT, L'Université et la crise ucomptable”. . . . . 317

\section{Comptes rendus}

Benoît LEMAIRE, L'espérance sans illusions. L'espérance chrétienne dans la perspective de Gustave Thibon, par Claude Gagnon. 337

Julien NAUD, Unephilosophie de l'imagination, par Claude Giroux. 340

COLlECTIF, Nation, Souveraineté, Draits. Actes du lVe Colloque interdisciplinaire de la Société de Pbilnsopbie du Québec: la question nationale, par Josiane Boulad Ayoub. . . . . . . . . . . 342

MARC CHABOT, Cbroniques masculines, par Marc Turgeon. . . 344

Normand LACHARITÉ, Le modele ER. Un système de catégories destiné à l'analyse des entreprises de recherche, par Maurice Lagueux.

Jacques CROTEAU, L'bomme: sujet ou objet? Prolégomènes philosophiques à une psychologie scientifíco-humaniste, par François Tournier. . . ............................. 351

Mare RENAULT, Le singulier. Essai de monadologie, par Léo-Paul Bordeleau. ......................... 355

Rober LEBEUF, Cosmic Presence, par Georges Hélal. ........ 359 Josiane AYOUB et al., Les épiphanies idéologiques, par André Paradis. . . . . . . . . . . . . . . . . . . 362 


\section{COMITE DE REDACTION DE LA REVUE}

Maurite Gagnon (Université de Sherbrooke), direcreur et responsable de la rubrique "Articles*. Josiane Boulad-Ayoub (Universiré du Québec à Montréal), direcreur adjoint et responsable de la promotion de la Revue.

Franşois Latraverse (Universirte du Québec à Montréal), responsable des rubriques * Érudes cririques * et * Compres rendus *.

Pierre Laberge (Université d'Ortawa), responsable de la rubrique "Bulletin" .

André Paradis (Universicé du Québec à Trois-Rivières), responsable de la rubrique unterventions" .

Guy Bouchard (Universiré Laval).

Danièle Lerocha (Université d'Ortawa).

Georges Hélal (Université de Montréal).

Robert Hébert (Collège Maisonneuve).

Stanley French (Université Concordia).

Yvan Clourier (Collège de Sherbrooke).

\section{COMITE DE REDACTION DE LA REVUE}

Maurice Gagnon (Universiré de Sherbrooke), direcreur et responsable de la rubrique *Articles» . Josiane Boulad-Ayoub (Université du Québec à Montréal), directeur adjoint et responsable de la promotion de la Revue.

François Larmerse (Unıversité du Québec à Montréal), responsable des rubriques * Érudes critiques - et - Comptes rendus .

Pierre Laberge (Université d'Ottawa), responsable de la rubrique *Bulletinn.

André Paradıs (Université du Québec à Trois-Rivières), responsable de la rubrique «Interventions.

Guy Bouchard (Université Laval).

Danièle Lerocha (Université d'Otrawa).

Georges Hélal (Université de Montréa)

Stanley French (Universıré Concordia).

Yvan Clourier (Collège de Sherbrooke). 


\section{COMITE DE REDACTION DE LA REVUE}

Maurice Gagnon (Universiré de Sherbrooke), directeur et responsable de la rubrique "Articles» . Josiane Boulad-Ayoub (Universiré du Québec à Montréal), direcreur adjoint er responsable de la promotion de la Revue.

François Latraverse (Université du Québec à Montrtal), responsable des rubriques * Études critiques - et - Compres rendus - .

Pierre Laberge (Université d'Ottawa), responsable de la rubrique «Bulletinm.

Jean-Claude Simard (College de Rimouski), responsable de la rubriquc "Interventions" André Carrier (College de Lévis-Lauzon).

Danièle Lerocha (Universıcé d'Orrawa).

Georges Hélal (Universiré de Montréal).

Michol Despland (Université Concordia).

René Pellerin (College Laflèche).

Yvan Clourier (College de Sherbrooke)

\section{COMITE DE REDACTION DE LA REVUE}

Maurice Gagnon (Université de Sherbrooke), direcreur et responsable de la rubrique "Articles". Josiane Boulad-A youb (Université du Québec à Montréa), directeur adjoinc et responsable de la promotion de la Revue.

Francous Latruverse (Universite du Quebec ì Montrtad), responsable des rubriques - Études crieiques - et - Compres rendus -.

Pierre Laberge (Universiré d'Otrawa), responsable de la rubrique -Bul lecin-

Jean-(.laude Simard (College de Rimouskı), responsable de la rubrique . Interventions "

André Carrier (College de Lévis-Lauzon).

Danıèle Lerocha (Universice d'Orrawa).

Georges Helal (Université de Montreal).

Mıchel Despland (Unuversice Concordia)

Hene Pellerin (College Lafleche).

Yvan Cloutser (Collecge de Sherbrooke).

\section{COMITE DE REDACTION DE LA REVUE}

Maurıce Gagnon (Université de Sherbrooke), directeur et responsable de la rubrique "Artıcles". Josıane Boulad-Ayoub (Université du Québec à Montréal), directeur adjoint er responsable de la promotion de la Revuc.

François Latraverse (Université du Québec à Montréal), responsable des rubriques - Érudes eritiques - et - Comptes rendus -

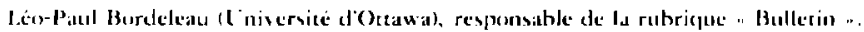

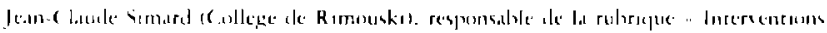

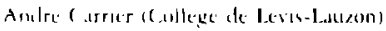

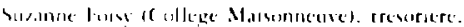

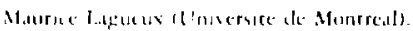

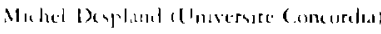

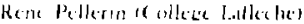

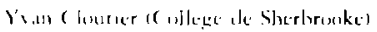


Volume $\mathrm{X}$, numéro 1

\section{SOMMAIRE}

Articles

A. PARADIS, Bernard-Henri Levy : le mal radical ou la philosophie du désespoir.

J. VUILLEMIN, Le chapitre $I X d u$ De Interpretatione d'A ristote. .......................................... 15

S. LAFLAMME, Sartre et la socioloyie. La notion de totalisation. .. 53

M. BOURDEAU, Grammaire et psychologie. Remarques sur la nature et les arigines de la grammaire générative.

Érudes critiques

R. HÉBERT, D'une falaise d'oì lon voit poindre le soleil de la culture (Le premier cahier de l'Instirut Québécois de Recherche sur la Culture) (Suire). ........................... 97

C. GAGNON, Défaire l'bistoire, de Réal Rodrigue............ 111

F. DUMONT, Une contribution à l'bistoire de la philosophie au Québec (La philosophie et son enseignement au Québec 1665-1920, par Yvan Lamonde).

\section{Interventions}

L. ChARETTE, R. IMLAY, R. CHAMPAGNE et $M$. RENAULT, Texte à plusieurs voix autour d'un livre (Le singulier. de Marc Renault).......................... 127

J.C. PETIT, La traduction françaist d'un outrage de H.G. Gadamer, Warheit und Methode.

Comptes rendus

Vianney DÉCARIE, Aristote. Éthique à Eudème, par G. Leroux. 177 John BURBIDGE, On Hegel's Logic. Fragments of a Commentary. par R. Lamberr................................ 180 Jacques DERRIDA, Lireille de l'autre, par M. Turgeon. ........ 182 Georges HÉLAL, La philosophie comme pan-physique. La philosophie des sciences de A.N. Whitebead, par S. Robert. .

\section{Livres reçus}

Avis aux lecteurs de Philosophiques et aux membres de la Société de Philosophie du Québec. 


\section{SOMMAIRE}

Volume $\mathrm{X}$, numéro 2

LE MARXISME CENT ANS APRĖS MARX

Présentation

L. P. Luc, Révolution et spéculation chez le jeune Marx.

J. Boulad Ayoub, La leçon de L'Idéologie Allemande.

Y. Cloutier, Gramsci et la question de l'idéologie

P. Ranger, Théorie de la connaissance, idéologie et camera obscura...

G. Bouchard, Marx, Bloch et l'idéologie

G. Raulet, Marxisme et condition post-moderne

M. Lagueux, Grandeur et misère du socialisme scientifique

ARTICLES

V. Mendenhall, Essai sur l'incompétence esthétique.

F. Giroux, Concept d'injustice sociale et absence d'intégration d'une classe en société civile

S. Labson, Dialogue sur l'infinité et la réalité

INTER VENTION

R. Houde, Genre et tendances. L'essai : sous-ensemble d'un ensemble 403

\section{COMPTES RENDUS}

Hilaire de Poitiers, La Trinité, traduction française de Mgr. Albertus Martin. par Claude Gagnon .......................

Mostafa Faghfoury, ed., Analytical Philosophy of Religion in Canada, par Robert Hébert 


\section{SOMMAIRE}

Volume XI, numéro 1

Avril 1984

Articles

J -P. MARgoT, La lectwre foucaldienne de Deriartes : sex présupposés et ses implications

1. HOULAD AYOUB, Limage du centre et la notion de l'un dans les

Ennéades

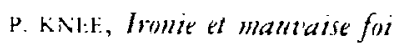

D. LAL'RI:R, Remarques sur la sémintique

Égalité, justice et différence

Nitte de las rédaction

I. Marcil l.aroste, Introduction générale : l'impasse dés égaux ......

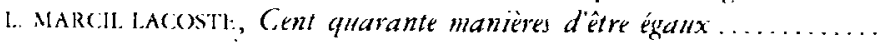

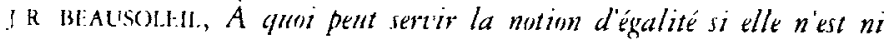
trament heuristique ni simplement éristique ?................

I SAINT-ARNALID, Les définitions aristotéliciennes de la justice : leurs rupports à la notuon d'égalité

\section{Interventions}

1.. Villomaire, Les chiens de garde de Panl Nizan

G. colvin, Pour un bilan culturel du Nouveau Monde...

\section{Étude critique}

R. HÉBERT, Sans trop mächer les mots, percevoir. Contribution au Réjean Ducharme, Nietzsche et Dionysos de Renée Ledur-Park....

\section{Comptes rendus}

SERGE ROBERT, Les révolutions du savoir, par Andrew Lugg et Donald McDonell

GUY H. ALLARD et SERGe LUSIGNAN, éditeurs, Les Arts mécaniques au moyen âge. par Robert Nadeau.......................

GÉRARD RAULET, Humanisation de la nature, naturalisation de l'bomme, Emst Blach ou le projet d'une autre rationalité, par Jacques $G$. Ruelland

gilles lane, À quoi bon la philosopbie ’. par René Pellerin ...... 213

Livres reçus. 


\section{SOMMAIRE}

Volume XI, numéro 2

Octobre 1984

Arricles

J. BOUlal) AYOUB, Descartes face à Leibniz sur la question de la substance ...........................................

R. MIGUELE\%, L'articulation du général et du particulier: une approche métbodologique dans le sbamp des sciences sociales...

F lepage, La naissance de la théorie des types.

U SANTAMARiA et A. MANVILLE, Marx: entre l'idéalisme radical et lindividualisme anarchique.

\section{Égalité, justice et différence}

Note de la rédaction

(. cOHIER, Le rapport masse-élite comme modèle canonique de la dialectique sociale.

Y. CiAUTHII:R, Note sur la syntaxe et la sémantique du concept d'égalité.

I - 3 ROUMANES, Une alternatite à l'exclusion: la dialogie ......... 353

F ciroux, De la société cinile à l'État : l'irruption de l'inégalité .... 373

M. MOROSoli, Les atuatars de la différence.

\section{Étude critique}

G. THI:RIEN, Dominances. Essai de socinbiologie sur l'inégalité et la tromperie. de Claude Lagadec

\section{Comptes rendus}

1. BLANCHARD, Cabiers de recherche sociolngique. vol. I, seprembre 1983, "Connaissance et société ". Collectif du Département de sociologie, Université du Québec à Montréal.....

(i. LeRoux, Tragique et tragédie dans la tradition occidentale / Tragedy and the Tragic in Western Culture, de Pierre Gravel et Timothy Reiss, éditeurs. 


\section{SOMMAIRE}

Volume XII, numéro 1

\section{Articles}

V. DE NGUYEN, Approche épistémologique de la caractérologie selon le point

de vue de J. C. Pariente.

L.-P. BORDELEAU, Un nouveau paradigme : le corps sportif .......... 33

R. BOUCHARD, L'idéalisme est-il spiritualiste ?................ 53

J. MOLINo, Pour une bistoire de l'interprétation : les étapes de

l'berméneutique.

Aspects philosophiques du féminisme

Note de la rédaction

J. BOULAD AYOUB. Éros androgyne et logos philosophique.

L. POISSANT, Pour l'immobilisme?

L. GODARD, Pour une nouvelle lecture de la question de la femme : essai

à partir de la pensée de Jacques Derrida ................... 147

M. LAROCHE, La femme (dite barrée) selon l'apprache lacanienne....... 165

M.C. BROUSSON-ROSAY, Psychanalyse et féminité ............... 177

\section{Intervention}

P. DESJARDINS, Les loteries nationales : une exploitation malbeurtuse? 191

\section{Bulletin}

F. LATRAVERSE, Les études wittgensteiniennes au Canada : état de la recbercbe, 1970-1984.

Étude critique

M.-F. ARChambault, L'Amérique du Nord et la culture, de Michel Morin

\section{Comptes rendus}

R. NADEAU, La physiologie des lumières. Empirisme, modeles et théories, de F. Duchesneau. - F. FOURNIER, Fondements et étapes de la resherche scientifique en psychologie, de Michèle Robert et alii. - J.N. KAUF. MANN, Science, valeurs et rationalité, de R. Miguelez. - G. LEROUX, Graceful Reason, de L.P. Gerson (ed.). - Daniel Laurier, Pragmatisme et pensée contemporaine, de LuC Bégin et alii. - MARC TUR. GEON, Le procès de la métaphore, de Guy Bouchard. 


\section{SOMMAIRE}

Volume XII, numéro 2

Articles

L.-P. BORDELEAU, Un nouveau paradigme : le corps sportif (suite)

J. MOLINO, Pour une bistoire de l'interprétation : les étapes de l'herméneutique (suite)

H.-M. GICQUEL, Le livre sur Adler de Soren Kierkegaard...... 315

D. GOLDSTICK, Lecture d'Altbusser........................ 363

\section{Interventions}

Entrevue avec M. Matthew Lipman

F. DUHESNEAU, Figures de l'empirisme. Discours de réception à la Sociéré Royale du Canada.

\section{Études critiques}

y Cloutier, Objet pour la philosopbie, de Marc Chabot et André Vidricaire (éds.)

M LA CHANCE, Le Prince, de Nicolas Machiavel, présentation et traduction par G. Allard; Le Prince et autres écrits politiques, de Nicolas Machiavel, présentation par P. Ranger, traduction par P. Ranger et J.V. Périès. 429

\section{Comptes rendus}

J. Y. SIMARD, Éloge de l'homme inutile, et Diversions. Huit opérations poétiques pour une stratégie métaphysique, et Veilleurs de nuit, de Alexis Klimov. - A. CARRIER, Si les marionnettes pouvaient choisir, de Gilles Lane. - MAURICE lagueux, Socialisme, Étatisme et Démocratie, de Dorval Brunelle. - MICHEl DUFOUR, L'anthropologie d'Althusser, de Donald Martel. 


\section{COMITE DE REDACTION DE LA REVUE}

Claude Savary (Université du Québec à Trois-Rivières), directeur et responsable de la rubrique * Articles *

Adèle Chené (Université de Montréal), directrice adjointe et responsable de la promotion de la Revue.

François Latraverse (Université du Québec à Montréal), responsable des rubriques "Etudes critiques* et *Comptes rendus .

Léo-Paul Bordeleau (Université d'Orrawa), responsable de la rubrique « Bulletin*.

Jean-Claude Simard (Collège de Rimouski), responsable de la rubrique a Interventions *.

André Carrier (Collège de Lévis-Lauzon).

Suzanne Foisy (Collège Montmorency), trésorière.

Maurice Lagueux (Universiré de Monrtéal).

Michel Despland (Université Concordia).

René Pellerin (Collège Laflèche).

Yvan Clourier (Collège de Sherbrooke).

\section{COMITE DE REDACTION DE LA REVUE}

Claude Savary (Université du Québec à Truis-Rivières), directeur et responsable de la rubrique "Arricles".

Adèle Chené (Université de Montréal), directrice adjointe et responsable de la promotion de la Revue.

François Lepage (Université de Montréal), responsable des rubriques, * Etudes critiques * et * Compres rendus *

Léo-Paul Bordeleau (Université d'Ottawa), responsable de la rubrique * Bullerin *.

Jean-Claude Simard (Collège de Rimouski), res ponsable de la rubrique * Interventions*.

André Carrier (Collège de Lévis-Lauzon).

Chrisrine Coallıer, trésorière.

Georges A. Legault (Universiré de Sherbrooke)

Michel Despland (Université Concordia)

René Pellerin (Cullège Laflèche).

Pierre Cohen-Bacrie (Collège Montmorency)

\section{COMITE DE REDACTION DE LA REVUE}

Claude Savary (Universiré du Québec à Trois-Rivières), directeur er responsable de la rubrique * Arricles *

Jocelyne Couture (Universiré du Québec à Montréal), directrice adjointe et responsable de la promocion de la Revue

François Lepage (Université de Montrécal), responsable des rubriques, * Études critiques * et "Comptes rendus $*$.

Diane Simpson (Université d'Orsawa), responsable de la rubrique * Bulletin *.

Pierre Cohen-Bacrie (Collège Montmorency), responsable de la rubrique * Interventions *. Jean Grondin (Universite Laval).

François Tournier (Université Laval), trésorier.

Georges A. Legault (Université de Sherbrooke).

Stanley French (Université Concordia).

Romain Gagné (Collège Garneau).

Claudette Lafond (Collège Marie-Victorin) 


\section{COMITÉ DE REDACTION DE LA REVUE}

Claude Savary (Universicé du Québec à Trois-Rivières), directeur et responsable de la rubrique \& Articles".

Jocelyne Couture (Université du Québec à Montréal), direcrrice adjointe et responsable de la promorion de la Revue.

François Lepage (Université de Montréal), responsable des rubriques, «Érudes critiques » et "Comptes rendus".

Diane Simpson (Université d'Ortawa), responsable de la rubrique "Bulletin *.

Pierre Cohen-Bacrie (Collège Montmorency), responsable de la rubrique «Interventions *. Renée Bilodeau (Universiré du Québec à Trois-Rivières), trésorière.

Jean Girondın (Université Laval).

Georges A. Legaulr (Université de Sherbrooke).

Stanley French (Universiré Concordia).

Romain Gagné (Collège Garneau).

Claudette Lafond (Collège Marie.Victorin).

\section{COMITÉ DE RÉDACTION DE LA REVUE}

Claude Savary (Université du Québec à Trois-Rivières), directeur et responsable de la rubrique - Articles *.

Jocelyne Couture (Université du Québec à Montréal), directrice adjointe et responsable de la promotion de la Revue.

Michel Scymour (Université de Montréal), responsable des rubriques * Études critiques - et - Comptes rendus.

Diane Simpson (Université d'Ottawa), responsable de la rubrique *Bulletin.

Pierre Cohen-Bacrie (Collège Montmorency), responsable de la rubrique Interventions :

Renée Bilodeau (Université du Québec à Trois-Rivières), trésorière.

Jean Grondin (Université d'Ottawa).

Georges A. Legault (Université de Sherbrooke).

Stanley French (Université Concordia).

Romain Gagné (Collège Garneau).

Philip Knee (Université Laval). 


\section{SOMMAIRE}

Volume XIII, numéro 1

\section{Articles}

(i. PRONOVOST, L'expression et les frontières de la réalité ..........

S. l.Aflamme, Problématique de philosopbies morales occiden. tales

J BOULAD AYOUB, $l l$ faut être idéologiste pour seulement en avoir l'siée...

1) JACQUETTE, Émergence et incorporation d'après Margolis.........

\section{Bulletin}

1. MARCII-LACOSTE, L'essai en philosophie: problématigue pour l'établissement d'un corpus .................................

\section{Interventions}

K NIFLSIN, La longue marche à travers les institutions: de Wiltgenstein à Marx. Allocution présidentielle au $28^{\circ}$ Congrès de l'ÁCP, juin 1984

R NADEAU, Hommages à Hugues Leblanc, philosophe logicien....

\section{Etudes critiques}

G. RAULIIT, Un bilan du marxisme?', à propos de : Le marxisme des années soixante - Une saison dans l'hirtoire de la pensée critique, de Maurice Lagueux.

M l.AGULUX, Un «bilan» vite parcouru (réponse à $G$. Raulet) .....

F. TOURNIER, Genèse du matérialisme dans les écrits de jeunesse de Karl Marx, de Jean-Guy Meunier.

J.G MEUNILR, Sur le matérialisme du jeune Marx (réponse à F. Tournier).

M. Lagiluux, L'idéologie et les stratégies de la raison, de Claude Savary er Claude Panaccio (dir.).

Compres rendus

cilinoux. L'urcbitecture de l'univers intelligible dans la pbilosophie de Plotin. Une étzde analytique et bistorigue, de A.H. Armstrong (craduction par Josiane Boulad Ayoub). - W. MOSER, et DANIELF LeTocha, The Sartre-Camus Controversy. A Literary and Pbilo-
sophical Critique, de Peter Royle. - F Lepacie, La logique - une introducteon, de Michel J. Blais. - E vol.ANT. Theories et pratiquer de la désaliénation, de René Pellerin.................

Remerciemenrs à nos lecteurs 


\section{SOMMAIRE}

Volume XIII, numéro 2

Articles

J C. CLAVET, Le concept de liberté chez Herbert Marcuse .......... 209

C. PANACCIO, Les qualités selon Stout........................ 237

V. DE NGUYEN, La critique des antbropologies et le Discours sur

l'inégalité de J.J. Rousseau............................. 253

R. TREMBLAY, Critique de la théorie marxiste de l'Etat............ 267

C. PICHE, Les fictions de la raison pure ......................... 291

S. PLOURDE, Du jeu et du sommeil à la gravité et à l'insomnie...... 305

\section{Interventions}

J. MARGolis, Pour réhabiliter la notion d'incorporation des entités culturelles : réponse à Dale Jacquette.................... 333

M. BUNGE, Culture et inculture ............................ 347

R. NADEAU, Contre le scientisme. Pour l'ouverture d'un nouveau front................................................... 353

Etudes critiques

M LA CHANCE, $l l y$ a toujours un mort celé dans le miroir : structure spéculaire et métaphores tragiques dans $D^{\prime u n}$ miroir et de quelques éclats de P. Gravel .......................... 369

J-G. MEUNIER, La logique illocutoire: Ses fondements selon Searle et Vanderveken, à propos de Foundations of Illocutionary Logic de J. Searle et D. Vanderveken......................

Comptes rendus

G LEROUX, Le Québec face à la formation morale, de G.A. Legault et L. Bégin; Les parents et le statut confessionnel de l'école au Québec, d'Anita Caron et collaborateurs..................... A.P ROSE, Philosophie et éducation, sous la direction de M. Gagnon er G. Legault ......................................

M. Renault, Certitudes et questions de la raison philosophique, de J. Theau ............................................ 414

Y SIMARD, L'utopie aujourd'bui, de G. Bouchard, L. Giroux et G. Leclerc ......................................... 422

Y. ClOUTIER, Autour de Jacques Lavigne, philosophe, de J. Beaudry 429 


\section{SOMMAIRE}

Volume XIV, numéro 1

Printemps 1987

Mort de l'archiviste

\section{Présentation}

G. LEROUX, Le sujet du souci : $A$ propos de l'Histoire de la sexualité de Micbel Foucault........................................ M. LA CHANCE, Déploiement et résistances chez Foucault..........

Articles

A. PARADIS, Pouvoir de la culture et culture du pouvoir...........

G. BOUCHARD, La métaphore heuristique de l'esclavage dans les textes féministes.....................................

\section{Interventions}

L'antbropologie des sciences: un programme pour la philosophie? (Entrevue avec Michel Serres).

C. PANACCIO, La responsabilité de la clarté.....................

\section{Etudes critiques}

N. LACHARITÉ, Dialectique et société, t. I: Introduction à une théorie générale du symbolique, et t. II : Culture, pouvoir, contrôle. Les modes de reproduction formels de la société, de Michel Freitag.........................................

M LA CHANCE. Sédiments 1986, de Georges Leroux et Michel Van Schendel (dir. de publication)........................

\section{Comptes rendus}

C. GAGNON, Jean Scot, écrivain: Actes duIV colloque international de la SPES, de Guy-H. Allard et collaborateurs...............

J.C. SIMARD, Le phénomène IXE-13, en collaboration........... 208

(; LEROUX, The Education of Desire: Plato and the Philosophy of Religion, de M. Despland............................ 213

R. LAMBERT, Hegel, l'Esprit absolu, de Th. Geraets (dir. de publicacion). 


\section{SOMMAIRE}

Volume XIV, numéro 2

Automne 1987

Articles

D. SAUVE, Kant, le matérialisme et la psychologie rationnelle

r. ALLARD, Au-delà et en deçà de l'bomme social: les póles de la pensée de Rousseau .....................................

P. KNEE, Agir sur les coeurs: spectacle et duplicité chez Rousseau

D. I.AURIER, Les états intentionnels des créatures solitaires ........

Y. PROVENÇAL, Une analyse de la notion d'objectivité ..............

U. SANTAMARIA ET A. MAINVILIE, Marx et le matérialisme: sens et valeur de la première thèse sur Feurbach

\section{Intervention}

G. LEROUX, Expérience, communauté, institution. Réflexions sur la spécialisation de l'éducation morale au primaire

Érude critique

R. MIGUeleZ, Narration, connaissance et identité chez Paul Ricœur (à propos de Temps et récit 1, 2 et 3) ....................

\section{Comptes rendus}

R F MARINEAU, Le fondement religieux de la pensée de Jung, de Lucie Bonnette. - E. CHRETIEN, Appel à la justice de l'État, de Pierre du Calvet (Extrairs présentés par Jean-Paul de Lagrave et Jacques-G. Ruelland). - J.G. RUELLAND, De l'éthique à la bioéthique, de Danielle Blondeau (sous la direction de). - J.G. RUELLAND, L'intelligence artificieuse, de Michel Germain. - N. GAGNON, Religion et culture au Québec. Figures contemporaines du sacré, de Yvon Desrosiers (sous la direction de). - J.G. RUELLAND, La petite revue de philosopbie, vol. 8, $\mathrm{n}^{\circ}$ 1, (automne 1986). - P. RANGER, L'actuel et l'actualité, de Jean-Paul Desbiens. - A. Martineau, Marcuse : La révolution radicale et le nouveau socialisme. Essai de synthèse, de André Vachet 
Volume XV, numéro 1

L'énigme de Kripke

Note de la rédacrion

M Sl:Ymour. Les énoncés de croyance et l'énigme de Kripke..... 5

D. I.AURIER, Note sur le puzzle de Kripke ...................... 31

c. PANACCIO, La notion de croyance: une approcbe inscriptionnaliste 41

F. LEPAGE, La question des attitudes propositionnelles et les limites de la sémantique

Descartes : le Discours de la Méthode

Note de la rédaction

I. MARCIL.LACOSTE, L'héritage cartésien : l'égalité épistémique .....

A VIDRICAIRE, La vision comme procédé de communication dans le Discours de la Méthode

\section{Arricles}

a.b. MaRChand. Mimèsis et catharsis: de la représentation ì la dénégation du réel chez Aristote, Artaud et Brecht ..........

Y IROVENCCAL, Remarques sur la notion de liberté dans l'bistoire occidentale et sur son dépassement possible ................

J AUMETRE, Habermas et Althusser : critique de l'idéologie scientiste et critique de l'bumanisme idéologique ....................

Y. CI.OUTIER. Philosophie et marketing: Surtre à Montréal, mars 1946

Études critiques

J R BraUSOLEIL, Le tournant dans la pensée de Martin Heidegger, de Jean Grondin ....

(i. BUCCHARD, L'androgynie comme modèle hétéropolitique, à propos de L'un est l'autre. Des relations entre hommes et femmes, d'Élisabeth Badinter

Comptes rendus

Y. CLOUTIFR, La naissance de la psychanalyse à Montréal, Frayages,

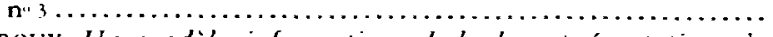
J. I.I:ROUX. Un modèle informationnel de la représentation, de N. Lacharicé . . . . . . . . . . . . . . . . . . . . . . . . .

A VIDRICAIRE, L'essai et la prose d'idées au Québec, sous la dir. de P. Wyczynski, F. Gallays et $\mathbf{S}$. Simard ............... 230

S. Fulsy, L'ceil et l'oreille, de M. Dufrenne ................. 234

R. Gervais, Herbert Marcuse's Utopia, d'Alain Martineau ...... 240 


\section{SOMMAIRE}

Volume XV, numéro 2

Automne 1988

Articles

S. CANTIN, De l'illusion à l'espérance ....................... 251

D. MOGGACH, Pbénoménologie et dialectique du travail ......... 311

A. LECLERC, La théorie générale des modes verbaux dans les grammaires philosophiques de l'époque classique .......... 331

P. BERNIER, «Suivre une règle " chez Wittgenstein: un paradoxe sceptique pour Saul Kripke ...............................

J. DE NGUYEN, Du Discours sur l'Inégalité à l'Emile : le parcours anthropologique de Roussean .............................

R. VALLEE, Le problème de l'esprit d'aut rus. Discussion de quelques solutions récentes

\section{Intervention}

L. MARCIL-LACOSTE, Du sens commun à l'égalité. Discours de réception à la Société Royale du Canada

Erude critique

V. MENDENHALL, Introduction à la méthodologie de la pensée écrite, de Normand Lacharité

Comptes rendus

J.G. RUELland, Histoire des sciences au Québec, de L. Charcrand,

$R$. Duchesne et $Y$. Gingras

J.G. Ruelland, Gaston Bachelard. Profils épistémologiques, de

G. Lafrance (dir de la publication)

S. PLOURDE, Immanence et transcendance chez Teilhard de Chardin, de $\mathrm{N}$. Bonner

P. BL.ACKBURN, Le laboureur et ses enfants: deux essais sur les limites de la rationalité, de J. Elster

A CARON, Foi en soi et confiance fondamentale. Dialogue entre Marcel Légaut et Erik H. Erikson, de J.-C. Breton ........... 


\section{SOMMAIRE}

Volume XVI, numéro 1

\section{Articles}

G. VACHON, Un conseil de Freud aux philosophes

G. GAUTHIER, L'engagement psychologique dans la communication langagière

D. SAUVE, Le moi-substance: une interprétation de l'a analyse du morceau de cire » de la seconde Médication

N. LACHARITE. Un modèle général pour décomposer la relation d'impact dans les recherches sur l'impact social de la science et de la technologie

\section{Intervention}

L. KERWIN, Les contributions de la science à la philosophie au $X X^{*}$ siècle

Etude critique

G. BOUCHARD, Du sexisme à la philosophie du sexisme. A propos de La raison en procès de Louise Marcil-Lacoste .............

L. MARCIL-LACOSTE, L'art du malentendu: une réponse à Guy Bouchard

\section{Comptes rendus}

F. DUGRE, Recherches sur le premier Aristote (Eudeme, De la philosophie, Protreptique), de B. Dumoulin .................. J. BEAUSOLEIL, Fragments de la période de Beme (1793-1796), de G.W.F. Hegel

$\boldsymbol{\gamma}$. CAutrhier, Cabiers de l'Ecole des Sciencer Pbilosopbigkes et Religieuses, 2-1987, publiés par les Facultés Universitaires Sainr-Louis . . . . . . . . . . . . . . . . . . . . . . . . . . . . . . . . M. CACNON, Etbique ex politique. Des valewrs personnelles a lemigement rocial de A. Morazain ec $S$. Pacella ............

R. RODEUS. Les Grecs el le desir de leete. Des Preplatoniciens a

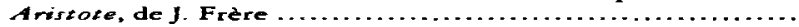

P. MICHAUD. Les jardins de Saine-Angusein : Lecrure des Confessions, de G. Tavard . ........................

M. DESPLAND. Persirtance et metamorphose de sacte. Actualiser Durkbeim el repenser la modernité, de J.A. Prades .........

G. LAFLEUR. La pensé de Karl Popper et la science economigue. numéro spécial de la revue Ëcomomie ex Sociésés, de A. Boyer

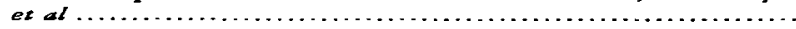
S. ROBERT. La sémantique dans les sciences physiques, de J. Leroux 


\section{SOMMAIRE}

Volume XVI, numéro 2

Automne 1989

\section{Arricles}

C.- (; GANCil:R, Les conditions proto-logiques des langues natu-

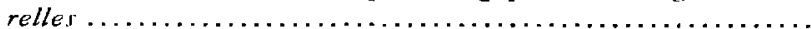

F. TOURNIER, La fondation de la bioétbique : une perspective épis-

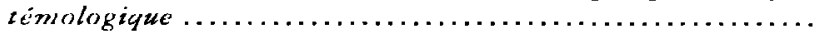

F. BlAIS, Réformisme pénal et responsabilité: une étude philoso-

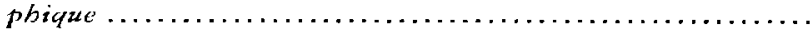

\section{Interventions}

I PESTIEAU, Quel développement et pour qui? ...............

A VOHOSAHI, Entre figures de la philosopbie: réflexion sur les

Figures de la philosophie québécoise ...............

R POllin, Savnir et croire. Sur le Pen et autres menus détails....

\section{Bulletin}

Y ClotITER, Sartriana québécoive: Chronologie, bibliographie et médiagraphie commentées.

Étude cricique

M ciaciNON, Genève de la théorie cellulaire, de François

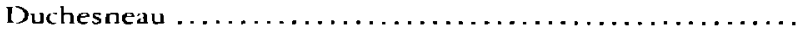

\section{Compres rendus}

Y. ROY, Pouvoir, jurtice et non-mépris, de Gilles Lane . . . . . . . .

I3 MI:RCIER, Autorité politique et liberté, de Yves Roy ............ M C.ACiNON, L'expérience et l'expression. Essai sur la pensée de

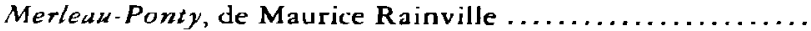

13 Rillix, Martin Headegger et Thomas d Aquin, de Johannic

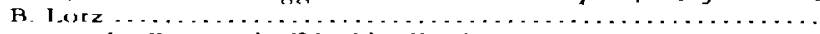

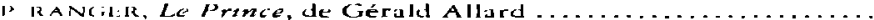

1. RANGIR, Vur unc redefinizien matérialiste du cancept do

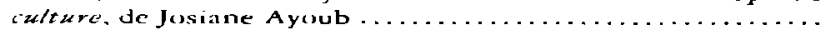

1" RANGil:R, Un modèle informationnel de la représentation, de

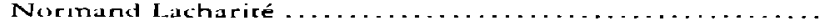

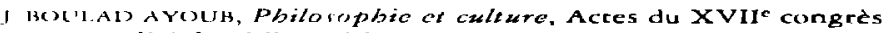
mondial de philosuphie .............................

I BOIIAD-AYUUH. Hiscoire de l'bistoire de la philosophie, de Mintial Civeruble . . . . . . . . . . . . . . . . . . . . . .

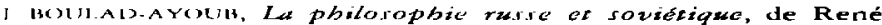

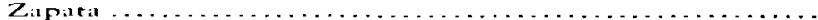

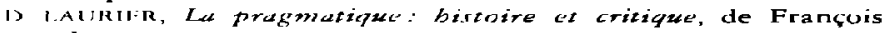

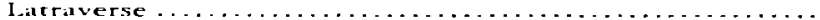

C. CIACINON, Figuret de la phalosopbie québécoise aprèr lex troubles

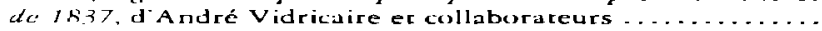




\section{SOMMAIRE}

Volume XVII, numéro 1

Printemps 1990

\section{Articles}

W.P. MENDONÇ, Intelligence artificielle et signification. A prapos des limites et des possibilités des sciences cognitives .........

G.A. LEGAULT, La parole du philosophe étbicien est-elle crédible?

D. SAUVE, Règles et langage privé chez Wittgenstein: deux interprétations .......................................

R. TREMBLAY, Analyse critique de quelques modèles sémiotiques de

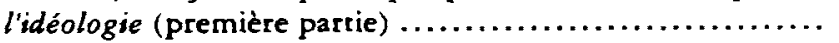

Note de recherche

P. KNEE, Le cercle et le doublet. Note sur Sartre et Foucault ......

\section{Intervention}

E. CHRETIEN, Entretien avec Monsieur Venant Cauchy ..........

\section{Etude critique}

M. FERLAND, Le silence des intellectuels. Radioscopie de l'intellectuel québécois, de Marc Henry Soulet

\section{Comptes rendus}

L.A. DORION, Les Présocratiques: bibliographie analytique (1879-1980), de Y. Lafrance, L. Paquet et M. Roussel ........

R. BODÉUS, Analyse génétique de la Métaphysique d'Aristote, de

Bertrand Dumoulin ....................................

R. BODEUUs, Justin. L'itinéraire philosophique, de E. Robillard ....

Y. GAUTHIER, Le théorème de Gödel, traduction de Jean-Baptiste

Scherrer ...........................................

A. VALEVICIUS, La notion d'accident chez Aristote, d'Alban Urbanas

J.G. RUelland, Machinations, de Louise Poissant ..............

J.G. RUELLAND, Les métamorphoses de la pensée libérale, sous la

direction de Lizette Jalbert et Lucille Beaudry ............ 162

G. Boss, Patience du Détachement, de Michel Cornu ........... 164

Y. GAUTHIER, Introduction à la logique, de François Rivenc...... 165

Livres reçus 


\section{SOMMAIRE}

Volume XVII, $\mathrm{n}^{\circ} 2$

Automne 1990

\section{Articles}

J. BOULAD-AYOUB, -Et la religlon le remplit de fureur... - Les déterminations tdéologiques, polémiques et poltitiques du Mabomet de Volcatre. . . . . . . . . . . . . . . . . . . . .

I. POLnKov, L'explictte et l'implicite dans la conception du signe chez Hobbes . . . . . . . . . . . . . . . . . . . .

R. TREMBLAY, Analyse critique de quelques modeles sémtotiques de l'tdélologie (deuxième partie). . . . . . .

M. MATtE, Jean-Jacques Rousseau et Friedricb Scbiller: Ie théatre sous le feu des lumidres.

J.C. ZEPHIR, Nature et fonction de la mémotre dans A la recbercbe du temps perdu ...............

Note de recherche

A. MORIN et J. EVERETT, Consctence de sot et langage intérteur: quelques spéculations.

\section{Étude critique}

s. FOISY, Homo Communtcativus: à propos de Luc Ferry, Homo Aestbeticus. L'invention du goút d l'age

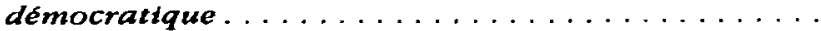

\section{Comptes rendus}

C. PANACCIO, Quatre introductions a la pbilosopbie au XIIT sidcle. Textes critiques et etude bistorique, de C. Lafleur. . . . . . . . . . . . . . . . . . . . . .

D. DUMAS, Le trancendantal et la pensée moderne. Études d'bistoire de la pbilosopbie, de A. Philonenko . . . . . . .

P. KNEE, Contre nous de la tyrannte... des relations tdéologiques entre Lumières et Revolution, de J. Boulad-Ayoub.

J. BOULAD-AYOUB, Dictionnatre bistorique de la Révolution françatse, d'Albert Soboul. . . . . . . . . . . . .

E. KATTAN, Les drotts de l'bomme et le drott nature, de Blandine Banet-Kriegel. . . . . . . . . . . . . . .

M.C. ClOZEL, Écrits de logtque pbilosopbtque, de Bertrand

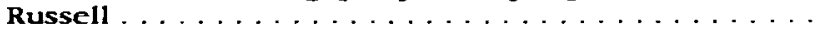

D. FISETTE, La pbilosopbie comme science rigoureuse, de Edmund Husser1. . . . . . . . . . . . . . . . . . .

C. PICHE, Hetdegger, de Alain Bontar. . . . . . . . . . . . . .

J.G. RUELLAND, La hberce. Libre volomes et maftrise de sot. de Claude Paris. . . . . . . . . . . . . . . . . . .

JG RUELLAND, Le dxscours feligienx. Une refiexion. de Claude Paris. . . . . . . . . . . . . . . .....

J SAINT-ARNAUD, Avortemene Out/Non, propos recuelilis par Michel Burviana. . . . . . . . . . . . . . . . . 


\section{SOMMAIRE}

Vol. XVIII, numéro 1 ,

Printemps 1991

\section{Articles}

M. SEYMOUR, Les énoncés psycbologiques da la premiere personne et le probleme de la connatssance de sot. . . . . . . . . . . . .

S. CANTIN, L'bomme de Marx est-il un sujet individuel ou un être social? À propos de l'interprétation de Marx par Louts Dumont. . .

F. TOURNIER, Un retournement dans la pbilosopbie de la biologie de K. R. Popper. . . . . . . . . .

J.-L. GOUIN, Merleau-Ponty et le marxisme. Ou la difficulté de tolérer l'intolérable . . . . . .

G. BOUCHARD, Typologie des tendances tbéoriques du fémintsme contemporatn. . . . . . . . . . .

\section{Étude critique}

M. TURGEON, Dissonance. Nietzscbe d la limite du langage, de Claude Lévesque . . . . . . . . .

Comptes rendus

R. MIGUELEz, $A$ la recbercbe du sens / In searcb of meaning, Revue de l'Université d'Ottawa / University of Ottawa Quarterly . . . . . . . . .

A. LECLERC, Witgenstein's Early Pbilosophy, de Donald Peterson . . . . . . . . . . . . . . . .

M. IMBEAULT, Le langage Heidegger, de H. Henri Meschonnic ....................

M. MARION, Poincaré, Russell, Zermelo et Peano. Textes de la discussion (1906-1912) sur les fondements des matbématiques : des antinomies à la prédicativité, de Gerhard Heinzmann (éditeur). . . . . . . . . . . 


\section{SOMMAIRE}

Vol. XVIII, No2

\section{Articles}

P. KNEE, Universalisme et pluralisme chez Montesquieu

E. BOURNEUF, Référence et dénotation des termes scientifiques

F. BLAIS, Avortement, éthique sociale et positivisme juridique

D. FISETTE, Pensée, langage et perception.

B. BAUGH, De l'individu à l'histoire: l'authenticité dans les écrits de Sartre

J. BOULAD-AYOUB, Les idées politiques de d'Holbach et la Déclaration des Droits de 1789

\section{Intervention}

R. KLIBANSKY, L'université allemande dans

les années trente (notes autobiographiques).

\section{Études critiques}

A. VIDRICAIRE, L'Amérique française devant l'opinion étrangère 1756-1960. Anthologie, de Robert I Iébert

S. CANTIN, Dialectique et totalitarisme, de Richard Gervais

\section{Comptes rendus}

G. BELLAVANCE, Amour comme passion. De la codification de I'intimité

Ecological Communication, de Niklas Luhmann ....

G. MICHAUD, Derrida, numéro spécial de la Revue philosophique de la France ct de l'etranger, sous la dir. de Catherine Malabou

M. TURGEON, A quoi perse la litterature? Exercices de philosophic littéraire, de Pierre Macherey

D. FISEITE, Le problème de la genese dars la philosophic de Fussert, de Jacques Derrida ........ 184

F. GRAVEL, Vocabulaire d'esthetique, sous la dir. de Etienne Souriau ....................................... 188

M. IMBEAULT, Soi-mëme comme un autre, de Paul Ricoeur ............................................. 190

P. BEIRNIER, Representation et réalité, de Iilary Putnam

R. TREMBLAY, Le processus interprétatif. Introduction à la sémiotique de C. S. Peirce, de Nicole Everaert-Desmedt 


\section{COMTIE SCIENTIFIQUE INTERNATIONAL}

Antonio Hermosa Andujar (Universilé de Séville); Miguel Beniez (Universié de Séville); Jacques Bidet (Paris VII-Nanterre); Jean-Pierre Comelti (Paris); Maurice Cranston (London School of Economics and Politics); Frank Cunningham (University of Toronto); Gérard Deledalle (Université de Perpignan); Marcel Hénaff (University of Califomia at San Diego); Raymond Klibansky (McGill University); Colette Michael (Northem Ulinois University); Roland Mortier (Universié libre de Brurelles); Shigeki Tominaga (Université de Kyolo).

\section{COMUIE DE REDACTION DE LA REVUE}

Josiane Boulad-Ayoub (Univessité du Québec à Montréal), drectrice et respansable des rubriques - Artides, - Interventions - et - Bulletin .

Georges A. Legauk (Université de Shertrooke), diredeur-adjoinc et responsable de la promocion de la Revue.

Misthel Seymour (Universié de Montréal), responsable des rubriques - Etudes citiques - et - Comples rendus :

Jacques Aumàre (Universié du Québec à Montréal), trésorier.

Philip Knee (Universié Laval).

Allison Laywine (Universié MoGill).

\section{COMIE SCIENTIFIQUE INTERNATIONAL}

Antonio Hermosa Andujar (Université de Séville); Miguel Benilez (Université de Sévile); Jacques Bidet (Paris VII-Nanterre); Jean-Pierre Cometi (Paris); Maurice Cranson (London School of Economics and Politics); Frank Cunningham (l Iniversity of Toronto); Gérard Deledalle (Universicé de Petpignan); Pascal Engel (Ecole Polyechnique de Paris/CREA); Dagfinn Follesdal (Sundford University); Marcel Hénaff (University of Califomin at San Diego); Raymond Klibansky (McGill University); Colete Michael (Northem Illinois University); Roland Mortier(Universiné libre de Bruxelles); John O' Neal (Hamiłon College, Harnilton); Shigeki Tominaga (Université de Kyoto); Pliilippe Van Parys (Universié Caholique de Louvain).

\section{COMITÉ DE RÉDACTION DE LA REVUE}

Josiane Boulad-Ayoub (Université du Québec à Montréal), directrice et responsable des rubriques - Articles :, - Interventions - et - Bullctin .

Georges A. Legault (Universilé de Shertrooke), directeur-adjoint et responsable de la promation de la Revue.

Micl kel Seynour (Universiné de Moruréal), responsuble des rubrix[ues. Éndes critiques • e • Comples rendus.

Jaçuses Aunkìre (Unjversité du Québec ̀̀ Moniréal), trésorier.

Plulip Knce (Unviersilé Laval).

Allison Laywure (Unversité McGill). 


\section{COMTÉ SCIENTIFIQUE INTERNATIONAL}

Antonio l lemosa Andujar (Unıversité de Séville); Henri Adan (Hōcel-Dieu, Paris); Mıguel Benılez (Université de Séville); Jacques Bidel (P.snis VI-N'anterre); Jean-Piene Cometi (Aix-Marseille); Maunce Cranston (London School of Economics and Politics), Frank Cunninglum (University of Toronto); Gérurd Dekedalle (Universilé de Perpignin), Pascal Engel (École Polytedınique de Paris/CREA); Dagfinn Follesdial (Sundford Univensity), Gilles-Gaston Granger (College de France); Marcel I lénaft (University of California al Sin Diego); Raymond KJibunsky (McGill University); Jean-Louis Labarière (CNRS-El IFSS); Piere Livel (Aix-Mirsseille); Coletle Michnel (Norhem Illinois University), Roland Morier (Université libre de Bnuxelles); John O' Neal (Hamilton College, Havulton); Charles Porsel (CNRS), Shigeki Tominaga (Université de Kyoko); Philippe Van Parijs (Louvain-lisNeuve)

\section{COMTÉ DE RÉDACTION DE LA REVUE}

Josiane Boulad-Ayoub (I'niversité du Québec à Montréal), directrice et responsible des rubriques "Articless -, "Interventions - et - Bulletin ".

Michel Seymour (L Iniversité de Montué.ll), directeur-adjoint et responssable de la promotion de la Revue.

Louis-André Dorion (Université de Monlréal), responsalble des rubriques - Éudes critiques - et - Comptes rendlus .

Jacques Aumère (Université du Quélec à Monuréal), trésorier.

Philip Knee (Universilé Lavil).

Allison Laywine ([ Iniversilé McGill).

\section{COMIEE SCIENTIFIQUE INTERNATIONAL}

Antonio Hermosa Andujar (Université de Séville); Henri Aulan (Hôtel-Dieu, Paris); Miguel Benitez (Universiť́ de Séville); Jacques Bidet (Paris VII-Nanterre); Jean-Pierre Cometri (Aix-Marseille); Maurice Cransion (London School of Econornics and Politios); Frank Cunningham (University of Toronto); Gerard Deledalle (Université de Perpignan); Pascal Engel (École Polynechnique de Paris/CREA); Dagfinn Føllesdal (Standford University); Gilles-Gaston Granger (College de France); Marcel Hénaft (University of California at San Diego); Raymond Klıbansky (MCGill University); Jean-Louis Labamière (CNRS-EHFSS); Pieme Livet (Aix-Marseile); Colette Michael (Nonhem Illinois Universiry); Roland Monter (Université libre de Bruxelles); John O' Neal (Hamilcon College); Charles Porset (CNRS); Shigeki Tominaga (Universite de Kyoto); Philippe Van Parijs (Louvain-la-Neuve); Du Xiazozhen (Université de Pékin).

\section{COMTTÉ DE RẼDACTION DE LA REVUE}

Jasiane Boulad-Ayoub (Universitê du Québec à Montréal), direcurice et responsable des nubriques

- Articles -, - Interventions - et - Bulletin -

Michel Seymour (Universilé de Montréal), direacur-adjoint et responsable de la promotion de la Revue.

Louis-André Dorion (Université de Montréal), responsable des rubriques - Études critiques - et - Comptes rendus :

Jacques Aumètre (Iniversité du Québec à Montréal), trésorier.

Philip Knee (Université Laval).

Allison laywine (Université McGill). 


\section{SOMMAIRE}

Vol. XIX, $\mathrm{N}^{\circ} 1$

\section{Articles}

JEAN-PIERRE COMETTI, Raison, argumentation et légitimation. Habermas, Apel et les apories de la communication

JOSETTE LANTEIGNE, Quelques remarques sur le jugement ..................................................... 25

LEE C. RICE, La causalité adéquate chez Spinoza ..........45 JEAN-ERNEST JOOS, Banalité du mal et sens $d u$ devoir chez les administrateurs de l'extermination

CHRISTINE FAURÉ, Le statut du serment et de la promesse dans la Déclaration des Droits de 1789.

Intervention

JOHN O'NEAL, Le continuum corps-esprit et l'économie de notre être selon Bonnet

Études critiques

GEORGES LEROUX, Heidegger et Platon.

Le problème du nihilisme, de Alain Boutot

ROBERT HÉBERT, Savoir des juges et savoir des juristes. Mes premiers regards sur la culture juridique québécoise, de Christian Atias

\section{Comptes rendus}

G. LEROUX, La naissance de la raison en Grèce, sous la dir. de Jean-François Mattéi. 
L.-A. DORION, Positions de la sophistique, sous la dir. de B. Cassin

G. PELLAND, Politiques, femmes pouvoir - Éléments d'une théorie de l'otage, de Pierre Gravel

M. DUFOUR, Kant. Avant/après, de Jean Grondin

M. FERLAND, Le Système de la Nature, ou des lois du monde physique et du monde moral, de Paul-Henry Thirry d'Holbach

P. GRAVEL, Martin Heidegger: Éléments pour une biographie de Hugo Ott

Y. GAUTHIER, Inventer l'univers. Le problème de la connaissance et les modèles cosmologiques, de Luc Brisson et F. Walter Meyerstein

G. BOUCHARD, Expérience esthétique et ontologie de l'œuvre, de Danielle Lories

R. VALLÉE, Pragmatics. A reader, sous la dir. de Steven Davis

L. FAUCHER, La stratégie de l'interprète: le sens commun et l'univers quotidien de Steven Davis

Livres reçus 
Volume XIX, $\mathrm{N}^{*} 2$

Automne 1992

\section{SOMMAIRE}

\section{Une nation peut-elle se donner la Constitution de son choix?}

Numéro spécial sous la direction de Michel Seymour

La Question nationale: Introduction pak Michel Seymour

Premitre Partie Nation et Nationalisme

LUKAS SOSOE: LE CONTTACTUAUSME ET LA QUESTION DES NATIONALITÉS 29 JOCELYNE COUTURE: L'ART DE LA SÉPARATION................................. 41

CHRISTIAN DUFOUR: LA SOCIE்IE் DISTINCTE ..................................... 55

MICHEL SEYMOUR. QUELUUES ASPECTS POLITIQUES DE L'ANTI-INDIVIDUALISME

Deuxabme Partie Constitution et Pluraissme

DOMINIQUE LEYDET: PATRIOTISME CONSTITUTIONNEL

ET IDENTITÉ NATIONALE.

WILL KYMLICKA: Le IIDÉRALISME ET LA POLITISATION DE LA CUITURE ... 93

DANIEI WEINSTOCK: LIDÉRAUSME, NATTONAUSME

et PLuR alisme CULTUREL

JOSEE LEGAULT: LES DANGERS d'UNE Chante DES DROITS ENChÀSSÉE

POUT UN QUÉUEC INDEEPENDANT.

Troisitme PARTIE SECESSION ET LIBERAUSME

ALLEN BUCHANAN: LES CONDITIONS DE LA SÉCESSION 159

KAI NIELSEN: Le faRdeau de La PREUVE

GARY CALDWELL: LE QUÉHEC NE DOIT PAS SE DONNER UNE CONSTITUTION: IL EN A DÉJA UNE QƯIL AUIANDONNEILAIT Á SES MISQUES ET PÉRILS. 191

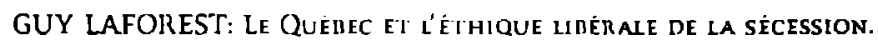
199 


\section{SOMMARE}

\section{CLAUDE GAUTHIER}

SPENCER, LE CONCEPT DE SOCIÉTÉ : ENTRE ORGANICISME ET INDIVIDUALISME ..... 3 DENIS SAUVE

Wittgenstein, Kripke et le paradoxe des Règles (I)

\section{PAISLEY IIVINGSTON}

LE DILEMME DE BRATMAN : PROBLĖMES de LA RATIONALITÉ DYNAMIQUE

\section{ANDRE PARADIS}

De Condillac $\dot{A}$ Pinel ou les fondements philosophiques dU traitement MORAL

\section{JEAN-LOUIS LABARRIERE}

Des Vertus DU DÉCENTREMENT dans LES RUtNes DE VOLNEY

\section{PIERRE JACOB}

FODOR, LA PSYCHOLOGIE SCIENTIFIQUE ET LES ATTRIBUTIONS DE CROYANCE..... 131

\section{INTERVENTION}

DOMINIQUE LECOURT : LES NOUVELES PHILOSOPHIES DE LA NATURE.

\section{ÉTUDES CRIIIOULS}

CHARLES PORSET : L'EFFICACITÉ DU SYMBOLIQUE.

Richard Caron : Gens de parole. Conférences publiques, essais ct débats à linstitut canadien de Montreal $1845-1871$, d'Yvan Lamonde

Suzanne Foisy : Correspondance $(1794-1802)$ de Fichte et Schelling. 
Yvon Gauthier : Le nombre et les nombres, d'Alain Badiou 204

Claude Gratton : Mircca Eliade : Io jour ef la nuit. Entre la litterature et la scicice. de Jacques Pierte. 205

Georges A. Legault : Introduction aux techniques de la philosophic, de Gilbert Boss 206

Georges A. Legault : Le paradigme biocthique. Une éthique pour la lechnoscience, de Gilbert Hottois. 208

Georges Leroux : Lhomme spéculaire, de Richard Rorty 209

Serge Leroux : Emile ou de l'́ducation, dans la nouvelle édition de Tanguy L'Aminot 213

Claude Pichè: Réduction et donation. Recherches sur Husscrl. Heidegger et la phénoménologie, de Jean-Luc Marion. .222

Sylvain Pinard : Claude Levi-Strauss, de Marcel Hénaff 224

Daniel M. Weinstock : Secession : The Morality of Political Divorce from Fort Sumter to Lithuania and Quebec, d'Allen Buchanan. 228

Daniel M. Weinstock : Léthique et les limites de la philosophic, de Bernard Williams 232 
Volume XX, $\mathbf{N}^{\prime} 2$

\section{NUMÉRO THÉMATIOUE

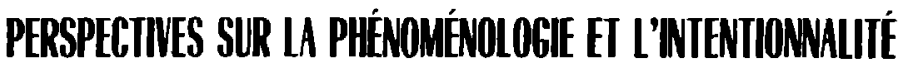

\section{SOMMAIRE}

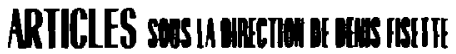

DENIS FISETIE : INTRODUCTION : LES SUITES DE LA PHÉNOMĖNOLOGIE.....247

HISSER, HEDEGER ET L'NTEITIONWUIIE

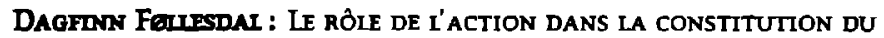
MONDE CHEZ HUSSERL ET HEIDEGgER

HUBERT DIFYFUs : AGIR, INTENTIONNAUTÉ ET ĖTRE-AU-MONDE

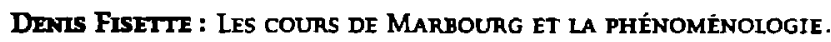
303

REALSSE, DEENSHE ET PRUEMTSME

Prerre livet : Structuhe nó́matioue et transcendance du Dasein ......323

Fabien Cayda : HuSSERL, Brentano et la pSychologie descruptTVE.

Dan Zafavt : Réduction et CONSTItution chez le Dernier HuSSERI.....363

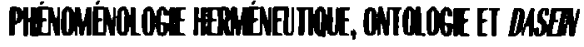

\section{JEAN GRONDDN :}

LA CONTRIBUTION SILFNCIEUSE DE HUSSERI À L'HERMÉNEUTIQUE. 383

PhIIP Bucterey :

LA NOTION D'AUTHENTICITÉ ChEz HUSSERI ET HEIDEgGER

STANLEY ROSEN : LA THÉORIE EXCÉDENTAIRE DE I'ÊTRE

Hentr AtLan: Projet et Signification dans des réseaux d'automates: LE RÓIF DE LA SOPHISTICATION 
NORYAND LACEARITE : VERS UNE ONTOLOGIE « NATURALISÉE 》

DE I'INFORMATION

LOUIS-ANDRE DORION : LA DIALECTIQUE D'ARISTOTE DÉNATURÉE

Renée Bilodeau : Paradoxes de lirrationalité de Donald Davidson

Antoine Cóté : L'autre Thomas d'Aquin de Martin Blais ..................................506

Louis-André Dorion : Introduction à la hétorique de Olivier Reboul............508

C. Dubois : Patriarcha ou le pouvoir naturel des rois, suivi des Observations sur Hobbes de Sir Robert Filmer.

Yvon Gauthier : Ie raisonnement par l'absurde de Jean-Louis Gardies ..........513

Yvon Gauthier : Ia vérfication de Gilles Gaston-Granger.............................514

Pierre Gravel : Shakespeare : les feux de l'enule de René Girard .........................516

Denis Sauvé : Wittgenstein. Les années de jeunesse : $188 g^{-1921}$ de Brian McGuiness ......................................................................518

André Vidricaire : Le Québec philosophique de Emile Chrétien ......................519

Vinh de Nguyen : Is nouvel ordre écologique. L'arbre, lanimal et lhomme de Luc Ferry

NDLR Philosophlques se rejoult de présenter a ses lecteurs le premier numéro de la sérle des numeros themati ques qu'elle inaugure avec cette livratson consacrée da la phénoménologie. Le numéro thématique alterne comme troistème numéro avec deux numéros réguliers; à part le corps d'articles à contenu libre. les rubriques habituelles sont maintenues.

M. Denis Fisette, professeur à IUQAM, a réunt les articles composant la partie thématique du numéro. Il assume la responsabilité de leur présentation et du travail d'édition. $M$. Fisette n'est pas parvenu à foumir les « abstracts » et les résumés qul accompagnent habituellement les articles dans Philosophiques. Quant au nombre de pages, le comité de rédaction a accepté qu'll dépasse les normes habituelles de la revue.

Monsleur Guy Bouchard, professeur à lUniversité Laval, sera le directeur de la publication du deuxième numero thématique. Ce numéro reprendra la plupart des contributions au XII colloque Interdisciplinaire de la SP.Q. qu'avait organisé Monsieur Guy Bouchard sur le thème des relations entre fermmes, litterature et philosophie. 